\title{
Los sentidos del embeddedness. Estudio comparado del concepto en la nueva sociología económica en Karl Polanyi
}

\author{
The senses of embeddedness. A comparative analysis \\ between the new economic sociology and Karl Polanyi's \\ approach
}

\author{
Truco, Ignacio; Locher, Valentina; Mariuti, Franco
}

Ignacio Truco ignacio.trucco@gmail.com (Universidad Nacional del Litoral - CONICET, Argentina

Valentina Locher mvlocher@gmail.com Universidad Nacional del Litoral - CONICET, Argentina

Franco Mariuti fa.mariutti@gmail.com Universidad Nacional del Litoral, Argentina

\author{
Estudios Sociales. Revista Universitaria Semestral \\ Universidad Nacional del Litoral, Argentina \\ ISSN: 0327-4934 \\ ISSN-e: 2250-6950 \\ Periodicidad: Semestral \\ núm. 61, e0002, 2021 \\ estudiossociales@unl.edu.ar \\ Recepción: 21 Julio 2020 \\ Aprobación: 01 Febrero 2021
}

URL: http://portal.amelica.org/ameli/jatsRepo/293/2932743002/ index.html

DOI: https://doi.org/10.14409/es.2021.2.e0002

El contenido está bajo Licencia Creative Commons AtribuciónNoComercial-Compartir Igual 4.0 Internacional. Atribución - No Comercial - Compartir Igual (BY-NC-SA): no se permite un uso comercial de la obra original ni de las posibles obras derivadas, la distribución de las cuales se debe hacer con una licencia igual a la que regula la obra original.

\section{c) (i) (2)}

Esta obra está bajo una Licencia Creative Commons AtribuciónCompartirIgual 4.0 Internacional.
Resumen: El término embeddedness, introducido por Karl Polanyi, se ha consolidado como una idea básica y constitutiva de la Nueva Sociología Económica (NSE). Sin embargo, la asimilación de este concepto introdujo diferencias significativas en relación a su significado original. Esta recepción creativa no siempre fue explicitada y en torno a ella se edificaron diferentes interpretaciones. En este trabajo se intentará contribuir a la clarificación de estas diferencias con el objetivo de lograr una mejor comprensión del término en cuestión, tanto en el desarrollo de la sociología económica contemporánea como en el pensamiento de Polanyi. Se argumentará que su recepción se basó en una adaptación de las nociones fundamentales de la sociología económica de Parsons y Smelser mediante el lenguaje de la Teoría de Redes y que ninguna de estas referencias sería compatible con la interpretación de Polanyi de los fenómenos socioeconómicos.

Palabras clave: racionalidad, utilitarismo, funcionalismo, economía sustantiva, integración económica.

Abstract: The term embeddedness, introduced by Karl Polanyi, has been consolidated as a basic and constitutive idea of the New Economic Sociology (NES). However, the assimilation of this concept introduced significant differences in relation to its original meaning. This creative reception was not always explicit and different interpretations were did. The propose of this paper is to contribute of achieving a better understanding of the term, in the contemporary economic sociology and in the Polanyi's thought. It will be argued that its reception was based on an adaptation of the fundamental notions of the economic sociology of Parsons and Smelser through the language of Network Theory and that none of these references would be compatible with Polanyi's interpretation of socio-economic phenomena.

Keywords: rationality, utilitarianism, functionalism, substantive economy, economic integration. 


\section{Introducción}

Desde la década de los 80 en adelante el concepto de embeddedness ha adquirido una importancia cada vez mayor en la sociología económica moderna, en especial dentro del campo denominado Nueva Sociología Económica (NSE). Dicho término, como es sabido, ha sido recuperado de los trabajos de Karl Polanyi, quien lo utilizara por primera vez en su obra La Gran Transformación ([1957] 2003:106 -de ahora en más LGT-).

Numerosas investigaciones han puesto en evidencia que el término embeddedness ha sido recibido de un modo creativo respecto del concepto original y ello ha contribuido a que, por un lado, la difusión e interpretación general del concepto sea confusa y, por otro, que el propio pensamiento de Polanyi sea mal interpretado.

En consecuencia, la relación entre la NSE y Karl Polanyi sigue siendo un problema no resuelto, no por la falta de investigaciones al respecto sino fundamentalmente por la dificultad que supone lograr una caracterización ajustada del pensamiento de Karl Polanyi, un pensamiento que no puede ser asimilado inmediatamente por las categorías usuales de la sociología económica.

Sobre la base de esta idea el trabajo se propone dos objetivos específicos:

- clarificar las diferencias que separan el concepto de embeddedness construido por la NSE y el concepto original desarrollado por Karl Polanyi,

- distinguir aspectos específicos del pensamiento de Polanyi que lo separan de la NSE en la constitución del objeto de estudio de la teoría social y económica.

A modo de hipótesis, se argumentará que la recepción creativa se basó en una reconstrucción del significado del término mediante el lenguaje de la Teoría de Redes. Sin embargo, por esta misma razón el concepto quedaría fuera del espectro teórico elaborado por Polanyi para analizar los fenómenos económicos. Por el contrario, se sostendrá que dicho autor se caracteriza por un enfoque en el que la realidad social es, en sí, una dualidad irreductible: una totalidad sociocultural, históricamente definida, con la capacidad de producir diferenciaciones cualitativas internas. Esta idea es directamente incompatible con la teoría del actor red. Mientras que esta última se aproxima a la totalidad mediante la acumulación de relaciones en una estructura reticular compleja, el sujeto social de Polanyi se inserta en las dos relaciones específicas e históricamente definidas: la comunidad y el individuo. Estas dos moralidades opuestas que se requieren entre sí le permiten forjar un objeto de estudio que queda completamente fuera del horizonte ontológico de la Teoría de Redes y de la NSE. En este marco, el término embeddedness y el estatus de lo económico como objeto de estudio, adquieren una significación no sólo diferente sino, aún, opuesta a la desarrollada por la NSE. 


\section{La nueva sociología económica y el embeddedness}

\section{La formación de la nueva sociología económica}

Podría observarse en Swedberg, Himmelstrand y Brulin (1987), sin mayores dificultades, la identificación de Economy and Society (1956) de Talcott Parsons y Neil Smelser, como el momento cumbre de la formación del campo de investigaciones científicas sobre la interacción entre lo económico y lo social.

Sintéticamente podría argumentarse que, desde Economy and Society hasta el presente, la sociología económica aceptó como punto de partida la siguiente demarcación: mientras la economía debía ser la encargada de estudiar el comportamiento racional de los individuos, la sociología tendría como propósito el estudio del comportamiento no racional de los individuos. Esta línea argumental se puede corroborar en los más conocidos manuales sintéticos de la NSE (Smelser y Swedberg, 2010; Granovetter y Swedberg, 2001).

Incluso Swedberg (1991) sugirió la existencia de tres escuelas del pensamiento en la sociología económica: la Escuela Alemana con sus orígenes en la escuela histórica y los trabajos de Weber, la Escuela Francesa con una fuerte influencia de las obras de Durkheim, y la Escuela Norteamericana con raíces en los trabajos de la Escuela de Chicago y de Talcott Parsons. Sin embargo, en el análisis de todas estas tradiciones Swedberg producirá lecturas confluyentes y compatibles con la separación entre la racionalidad utilitarista para la economía y la irracionalidad culturalista (a lo que podría agregarse la racionalidad con arreglo de valores) para la sociología.

Esto se puede ver con claridad en el trabajo de Swedberg sobre la sociología económica de Max Weber, donde establece esta distinción, indicando que la unidad de análisis básica tanto de la economía como de la sociología económica es la utilidad. Mientras que para el primer caso se trata de la utilidad sin referencia a otros, en el segundo es la utilidad definida en relación a otros y por lo tanto expuesta a las restricciones del hábito y la cultura (Swedberg, 2005:48).

Por su parte, el propio Mark Granovetter analizó la delimitación del campo y reconoció que el funcional-estructuralismo de Parsons no contaba con las herramientas conceptuales adecuadas para abordar la complejidad de dicha intersección.

Granovetter reconoció en Parsons la aceptación de la definición de Lionel Robbins acerca de la economía como una ciencia de la escasez y el rechazo del institucionalismo americano como definición del objeto de la teoría económica (aduciendo «insuficiencia analítica») (Granovetter, 1990:91). Pero, si bien aceptará esta distinción, lo hará bajo un conjunto de observaciones críticas que determinarán lo nuevo de la nueva sociología económica.

Para comprender las consideraciones críticas de Granovetter, conviene explicitar el modo en que estas articulaciones fueron originalmente interpretadas bajo un lenguaje general: el del funcional-estructuralismo y la teoría de los subsistemas sociales.

En este sentido, Parsons definió lo económico como un subsistema específico, o un caso particular, en el marco, más amplio, de subsistemas articulados, que compondrían a la sociedad en su conjunto. En este contexto teórico se produce el trabajo liminar de Parsons y Smelser (1956), donde estas hipótesis son 
desarrolladas y, luego, retomadas y sintetizadas por el propio Smelser en Sociología de la vida económica (1964).

En dicho trabajo Smelser afirma que es posible distinguir a la economía y a la sociología como disciplinas separadas y bien delimitadas. Para el caso de la economía, el autor se remite al propio Samuelson y a su definición de la economía como una ciencia de la escasez, donde se asume la existencia de una racionalidad utilitarista que cumpliría un rol regulativo, es decir, un como si de referencia en torno al cual la acción económica es evaluada. La sociología en cambio, tiene como campo de estudio al comportamiento del hombre en el marco de su relación con la estructura social, donde este término se entiende como el resultado de la «interacción repetida y regularizada entre dos o más personas» (Smelser, 1964:52). Por lo tanto, el horizonte de la sociología se definiría por los comportamientos orientados hacia sus semejantes.

La crítica central de Granovetter pasará por la capacidad de la teoría de los subsistemas para dar cuenta de las articulaciones entre el subsistema económico y el subsistema social. Según este autor, Parsons fracasó en captar la atención de los académicos de la economía, dado que no tenía aportes sustanciales o modificaciones relevantes que sugerir y, por lo tanto, no tenía nada que ofrecer a cambio de que ellos se interesaran en la sociología económica.

En definitiva, Granovetter observará que la teoría de subsistemas de Parsons y Smelser no resultaba de utilidad para abordar el verdadero problema no resuelto: la articulación entre la economía (como ciencia de la escasez) y la sociología (que tendría por objeto la totalidad de los subsistemas sociales). Faltaba, según el renovador, un eslabón que permita precisar estas relaciones (Granovetter, 1990:92).

En respuesta a este interrogante es que Granovetter postula a la NSE como la renovación teórica. Para ello introdujo dos elementos fundamentales: la teoría de redes y el concepto de embeddedness.

En este sentido, Swedberg observa los orígenes de la NSE en los trabajos pioneros de Harrison White (1981) sobre el análisis de mercados, el estudio comparativo sobre las diferentes redes dentro de un mercado de Wayne Baker (1984), la obra Economic Sociology de Arthur Stinchcombe (1983) y el artículo de James Coleman (1984) en el cual el autor «subraya la necesidad de tomar en cuenta las estructuras sociales al seno mismo del análisis económico» (Swedberg, 2001:7).

En todo caso, se produjo cierto consenso en torno a la importancia de los trabajos de Harrison White y sus discípulos (entre ellos Granovetter) como la puerta de para introducir a la teoría de redes como un lenguaje capaz de reconocer y delimitar el objeto de estudio de la sociología económica (Convert y Heilbron, 2007:37).

Sin embargo, el paso decisivo lo dará Mark Granovetter con The strength of weak ties (1973; y su versión ampliada de 1983) ${ }^{[1]}$. En este artículo, el autor introduce un nuevo lenguaje y un cambio de enfoque que pretende romper con el funcional-estructuralismo parsoniano, a la manera de una reestructuración.

Concretamente, Granovetter argumentó que la teoría de redes es el método más acertado para el estudio de las relaciones interpersonales, ya que representa un puente entre lo micro y lo macro. La falla en la que incurrieron los modelos anteriores, basados en los subsistemas, radicó, precisamente, en centrar 
su atención sólo en aquellos sistemas de relaciones sociales densas, en los cuales existen lazos interpersonales fuertes y visibles. Ello supondría un recorte arbitrario del campo de observación.

Frente a ello Granovetter propuso poner el énfasis en aquellas estructuras sociales menos visibles, los lazos débiles o menos densos, que son, a la vez, más fuertes, es decir, capaces de dar integración al sistema social y funcionar como un puente entre las relaciones interpersonales a nivel micro. Sintéticamente, los lazos débiles posibilitan el paso de las interacciones a pequeña escala a patrones de comportamiento a gran escala (Granovetter, 1983:1360).

De esta manera, Granovetter aseguró que, a través de la teoría de redes, las metáforas basadas en aparatos institucionales más o menos sólidos y estables, serán reemplazadas por sistemas de relaciones reticulares complejas y emergentes (propiedades básicas de una red según este enfoque), lo que haría posible conocer y comprender con mayor precisión el vínculo entre economía y sociología, donde la primera se refiere a las mencionadas relaciones interpersonales a nivel micro, mientras que la segunda remite a las relaciones macro.

Swedberg, al referirse la posición de Granovetter con respecto a la ciencia económica, diría que «el desplazamiento así operado por Granovetter abrirá la vía a un nuevo tipo de análisis, en los cuales supone simultáneamente que los actores son racionales y que las estructuras sociales juegan un papel: la sociología y la racionalidad pueden perfectamente coexistir» (Swedberg, 2001:8).

En una línea similar pueden ubicarse los aportes de otros investigadores que, por ejemplo, mostraron cierta continuidad y relación entre el programa de la NSE y el nuevo institucionalismo en el campo de la sociología (Nee e Ingram, 1992).

Finalmente, este programa se consolida con el artículo liminar que definirá la identidad de la NSE. Mark Granovetter publica en 1985, en la American Journal of Sociology, "Economic Action and Social Structure: The Problem of Embeddedness", en el cual situó el concepto de embeddedness como el término clave de la definición del objeto de este campo.

\section{El concepto de embeddedness en la nueva sociología económica}

Según el propio Granovetter el término embeddedness se asocia a la perspectiva sustantivista de Karl Polanyi, a la «economía moral» y el pensamiento de Marx (Granovetter, 1985:482). Sin embargo, el autor no explora con claridad estas relaciones conceptuales que, por cierto, introducen nuevas problemáticas.

Swedberg, por ejemplo, se remite al artículo mencionado para observar que el término embeddedness fue concebido como una alternativa a la noción de atomicidad (Swedberg, 2001:11). Así, la sociología económica tendría por objeto el encuadre o el incrustamiento de lo económico en lo social, es decir, el embeddedness mismo.

La interpretación de Swedberg puede ser incluso precisada volviendo sobre las hipótesis vertidas en Economic Action and Social Structure, en donde Granovetter definió dos posiciones polares acerca de los fundamentos de la acción económica. Según él, existen teorías que conciben al comportamiento económico de una manera infra-socializada (undersocialized) mientras que otra lo hace de un modo sobre-socializado (oversocialized). 
Granovetter asocia lo infra-socializada directamente a la teoría económica clásica y neoclásica (Granovetter, 1985:483) ${ }^{[2]}$, aunque, en rigor, es posible interpretar que Granovetter, con término infra-socializada, se remite, más específicamente al utilitarismo en el marco de la teoría neoclásica.

En cuanto a las teorías que tienen una concepción de la acción económica sobre-socializada, Granovetter las caracteriza de la siguiente manera:

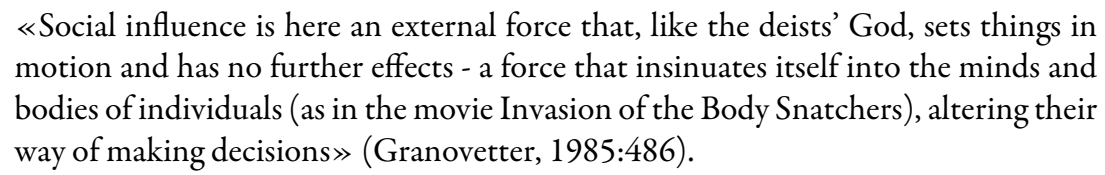
motion and has no further effects - a force that insinuates itself into the minds and bodies of individuals (as in the movie Invasion of the Body Snatchers), altering their way of making decisions» (Granovetter, 1985:486).

Sintéticamente, el comportamiento, en este tipo de teorías, quedaría determinado por el rol que la estructura social le asigna. Una vez que su lugar es determinado, el sujeto reproduce las normas y valores que la sociedad le imprime. En otras palabras, la sobre-socialización remite a la estructura social como única vía de socialización en la que se suprime la autonomía individual. El ejemplo que Granovetter utiliza es, precisamente, el de la teoría parsoniana-smelseriana de la sociología económica (Granovetter, 1985:486).

La noción de embeddedness que Granovetter propone es postulada como una superación de esta dicotomía. Se trata, como se argumentó hasta aquí, de ubicarse en un punto intermedio, en una gradación de relaciones reticulares. De este modo la racionalidad utilitarista sobrevive aunque rodeada de relaciones de densidad variable (Granovetter, 1985:487).

Como puede observarse, el concepto de embeddedness constituye, en definitiva, el objeto mismo de la NSE, a saber: la racionalidad utilitarista condicionada por relaciones interpersonales (de densidad variable) que presentan las propiedades identificadas por la teoría de redes (reticularidad, complejidad y emergencia).

Esta hipótesis, no sólo constituyó la base del desarrollo de la NSE desde mediados de la década de 1980, sino que además, permaneció de un modo más o menos inalterado hasta el presente. Granovetter publica en 2017 Society and Economy, y hace referencia explícita a esta situación e incluso advierte de una lectura estrecha del concepto de embeddedness, es decir, como un espacio de relaciones meso económicas. En rigor, tal y como lo indica Granovetter en este texto de síntesis, lo embebido de los sistemas económicos, se da en la articulación con otras esferas (sistemas) de la vida social.

El lenguaje de la teoría de redes, permitiría, según Granovetter, capar niveles diferentes de la realidad económica embebida: relacional (a nivel de los individuos y sus «funciones de utilidad interdependientes»), o estructural (a nivel super estructural, es decir, las «relaciones densas») (Granovetter, 2017:15-20). En medio, la meso articulación, supone una gradación infinita de posibles densidades de sistemas reticulares con propiedades emergentes.

\section{Las discusiones en torno al concepto de embeddedness en la NSE}

Además de las ideas principales que constituyen el núcleo de la NSE es posible distinguir un conjunto de autores que han realizado aportes críticos a la noción de embeddedness, tomando cierta distancia de Granovetter y que llevaron a la sociología económica más allá de los límites de la formulación arriba expuesta. 
Estos aportes pueden rastrearse, en particular en la década de los 90 y los 2000, y no son precisamente homogéneos. No obstante, tampoco es claro que ellos, en general, haya estado sistemáticamente preocupados por comprender el sentido original del término sino más bien, y eso se intentará mostrar a continuación, adaptar su significado a fin de captar aspectos de la realidad socio económica que el embeddedness de la NSE no parecería poder captar: las estructuras de clase, las relaciones de poder, la lucha entre estados, el papel de la etnicidad, entre otras dimensiones.

Por un lado, podemos observar ciertas críticas amistosas como la de Brian Uzzi, en Social Structure and Competition in Interfirm Networks: The paradox of Embeddedness (1997), quien propuso modificaciones al concepto de Granovetter a partir de un estudio empírico sobre veinte empresas de indumentaria realizado en New York, en donde distinguió relaciones mercantiles que pueden dividirse entre relaciones de mercado y relaciones especiales o cercanas. Estas últimas suelen darse en situaciones donde la confianza es importante, cuando se comparte información valiosa o en la resolución conjunta de problemas (Uzzi, 1997:63). En un sentido similar puede sumarse, una lectura también complementaria a la de la NSE. Roger Waldinger quien estudia el concepto de embeddedness observando cómo las redes étnicas permiten contener los efectos disgregadores del mercado, pero generan mecanismos de exclusión significativos contra los neófitos que quieren incorporarse en las redes de producción.

Sin embargo, por otra parte, es posible identificar un conjunto trabajos críticos de la recepción del término embeddedness por parte de la NSE basados una lectura alternativa que podemos caracterizar de abierta o no determinada, definida por la aceptación de una teoría relacional de la acción económica, aunque signada por la contingencia y la acumulación de relaciones específicas (la complejidad concreta) que crean una estructura emergente.

Jens Beckert, por ejemplo, analizó los cambios que ha sufrido el concepto embeddedness al ser reinterpretado por la NSE, poniendo especial atención en la lectura elaborada por Granovetter (Beckert, 1996). En este sentido, el autor señaló que la visión funcional-estructuralista adaptada al lenguaje de la teoría de redes, conlleva una «gran transformación» (Beckert, 2003 y 2007) del concepto original expuesto por Polanyi. Ello se observaría con particular claridad en la noción de mercado compuestas por uno y otro. Según Beckert, para Polanyi, los mercados serían instituciones sociales producto de un complejo entramado construido por la política, la cultura y las ideologías. Mientras que, para Granovetter, el mercado sería el resultado de comportamientos utilitaristas en el marco de una trama reticular de relaciones sociales. Esta última definición supondría dos dificultades simultáneas: una preferencia no justificada por la racionalidad utilitarista (como fundamento del intercambio) y una falta de explicación del origen de las estructuras sociales que producen los mercados específicos.

De este modo Beckert observó que, en el argumento de Granovetter, el embeddedness funciona como un conector entre aquellas conductas que pueden ser explicadas a través de la teoría del actor racional y los fenómenos observados en la realidad que no encuadran dentro del enfoque utilitarista. De modo que, dicho concepto, expresa sobre todo la debilidad del núcleo de la NSE y no su fortaleza (Beckert, 1999:3). 
Frente a esta situación Beckert se propone establecer una interpretación del concepto de embeddedness y de la acción económica que signifique un «quiebre radical» respecto de la tradición marginalista (Beckert, 1999:9). Para lograr esto el autor no abandona las nociones fundamentales de la teoría de redes (reticularidad, complejidad y emergencia) sino que, por el contrario, las afirma abandonando el egoísmo utilitarista como racionalidad de base. El comportamiento económico sería entonces, de un modo más general, el resultado de decisiones que los sujetos construyen bajo una trama de relaciones intersubjetivas con propiedades reticulares (Beckert, 1999:10).

En definitiva, Beckert sintetizó una interpretación del concepto de embeddedness bajo la fórmula de la estructuración social de la acción económica sin la necesidad de considerar una relación específica como determinante: «undetermined but not unstructured»(Beckert, 1999:4). Es decir, una interpretación abierta del concepto de modo tal que la racionalidad utilitarista puede ser parte del proceso, aunque no necesariamente.

Esto también puede verse en el trabajo de Sharon Zukin y Paul DiMaggio, quienes discutieron la noción de embeddedness propuesta por la NSE y el propio Jens Beckert observó que Structures of Capital (Zukin y DiMaggio, 1990) constituye una obra clave para lograr una conexión y complementariedad entre la NSE y su interpretación abierta de la acción económica (Beckert, 1999:31). En este caso, puede verse una pluralidad de modos de incrustamiento, que emergen, precisamente de la apertura en la que el enfoque se basa.

Para Beckert, el concepto de embeddedness estructural de estos autores es compatible con su propia definición del término. Sin embargo, estos adicionaron otras tres formas alternativas de incrustamiento (cultural, político y cognitivo) lo que introduce cierta ambigüedad en torno el significado del término en cuestión. Es difícil distinguir con claridad si se trata de racionalidades alternativas que se articulan entre sí, o si son diferentes dimensiones de un incrustramiento más general que abarca toda acción social.

Puede mencionarse, por otra parte, el caso de Viviana Zelizer quien también propone una interpretación abierta del término embeddedness aunque poniendo énfasis en la consideración los aspectos culturales que condicionan las redes de relaciones que estructuran la acción socioeconómica (Zelizer, 2008 y 2010).

Al igual que Beckert, la autora rechazó de plano la existencia de una racionalidad utilitarista primaria o fundamental. En consecuencia, si bien reconoció los aportes de la NSE considera que no logró superar los supuestos fundamentales del utilitarismo marginalista. La dimensión cultural de la vida económica permanecería eclipsada bajo el paraguas de la NSE (Zelizer, 1988:376-377).

Zelizer pretende encontrar una alternativa apuntando hacia la construcción de un modelo teórico, «that will explore and explain the complex historical, cultural, and social-structural variability of economic life» (Zelizer, 1988:379), pero sin caer en una dicotomía entre argumentos «culturales» y «socioestructurales» (Zelizer, 2008:100).

Un punto de vista convergente al de autores anteriores es el de Greta Krippner, quien observa la influencia de Parsons y Smelser en la recepción que Granovetter hace del término embeddedness y su alejamiento del pensamiento original de Polanyi. Con un argumento similar al desarrollado en este trabajo. 
Al caracterizar el pensamiento de Granovetter mediante la conservación del núcleo atomista del pensamiento neoclásico, al momento de referirse a lo propiamente económico, la autora repite, en términos generales la crítica de Beckert (Krippner, 2002: 786-7). La teoría de redes, que precisamente surge con el objetivo de captar la complejidad y la emergencia como concepción de lo social, estructurada pero no determinada (para usar la fórmula de Beckert) podría ser un marco adecuado para interpretar el embeddedness de Polanyi.

Krippner hace el intento de interpretar los conceptos principales de Polanyi al calor de esta lectura abierta del término, pero los resultados son poco estimulantes. En términos generales, la jerarquización de los conceptos abstractos (intercambio, reciprocidad y redistribución) sobre la caracterización histórica (mercado, hábitat, mercancías ficticias, doble movimiento), conduce a una suerte de vaciamiento de los atributos históricos que, para Polanyi, caracterizan a la sociedad moderna y su transformación en el tiempo. Krippner aborda la aparentemente «paradójica» expresión de Polanyi de que precisamente los mercados se hayan «desembebidos», argumentando que la obra del autor se estructura en diferentes niveles. Por un lado la caracterización del industrialismo británico del siglo XIX, proceso de autonomización del mercado, y por otro una concepción general de las instituciones económicas, en donde los mercado, incluso en su forma ideal «are not the expression of primal, timeless instincts; they are rather fully social institutions, reflecting a complex alchemy of politics, culture, and ideology» (Krippner, 2002:782). Esta separación en diferentes niveles de análisis, como se verá, se aleja significativamente del proyecto teórico del autor.

Por último, es posible considerar un punto de vista alternativo. El de Alejandro Portes quien puso el énfasis en las relaciones de poder y la estructura de clases (Portes, 2010). Portes se sitúa en el giro dado por Granovetter en 1985 y construye una lectura alternativa, basada en las relaciones de poder y propone un esquema de incrustación de las relaciones de clase en el marco de estructuras institucionales. El término embeddedness definiría precisamente la convergencia de dos campos cuya reconciliación no está precisamente resuelta en el análisis de Portes. La estructura de clases, por un lado, y los valores / normas, por otro, se articulan embebiéndose según el esquema de Portes, reemplazando de este modo las hipótesis utilitaristas por las categorías distributivas de la economía política clásica (Portes, 2010:69).

Como pudo observarse, las críticas a la NSE apuntaron fundamentalmente a la relación de ésta con el núcleo hipotético de la economía marginalista: la racionalidad utilitarista como racionalidad de fondo de las relaciones económicas. Sin embargo, en términos generales, las críticas realizadas a Granovetter y la NSE, no tuvieron como objetivo principal recuperar el pensamiento Karl Polanyi y su interpretación de la acción económica. Por el contrario, adaptaron el término embeddedness para otorgarle funcionalidad en esquemas de análisis propios: sea en la interpretación abierta o no determinada o aquella basada en las categorías fundamentales de la economía política clásica.

En particular, la idea de una complejidad abierta o no determinada como marco de análisis de la acción económica, no tiene demasiado sentido bajo la obra de un autor que, por el contrario, historizó su objeto de estudio de un modo radical y, como se intentará argumentar en el siguiente apartado, se apoyó en 
una fundamentación de su objeto de estudio definido como unidades histórico culturales capaces de producir diferenciaciones internas específicas.

\section{El embeddedness en la obra de Polanyi. Una lectura alternativa}

Como pudo observarse todos los autores de la NSE adoptaron, de alguna forma u otra, una interpretación no histórica de la acción económica. Determinadas (en última instancia, por la racionalidad utilitarista) en el caso de la NSE principal, o indeterminadas / abiertas en el caso de los críticos más radicales.

Por el contrario, el autor húngaro compondrá una interpretación de la acción económica basada en tres propiedades: primero, la sociedad es una unidad capaz integrar diferencias; segundo, esta unidad diferenciada supone un movimiento interno permanente; $y$, fundamentalmente, es de naturaleza histórica y no abstracta, de tal modo que sólo puede ser definida por su especificidad histórica.

Estas características separan radicalmente el pensamiento de Polanyi de las ideas de la NSE y de sus críticos. A continuación, se explicará con mayor detalle cada uno de estos elementos.

\section{El embeddedness en la gran transformación}

Para poder mostrar estos argumentos conviene indicar, en primer lugar, que el objetivo y la orientación general La Gran Transformación [1957] (2003) se distingue por una evaluación de la especificidad histórica del capitalismo moderno, su desarrollo en el siglo XIX y su crisis en el XX. Considerando estas características es más fácil observar los rasgos centrales del pensamiento de Polanyi y precisar el sentido que el término embeddedness adquiere en esta obra. No se trata de partir de relaciones formales que describen la circulación de mercancías, en tanto categorías generales y abstractas, para luego dar una interpretación a los diferentes momentos históricos del capitalismo, sino de partir de la especificidad histórica de su objeto y descubrir luego una fenomenología de su desarrollo.

Es posible distinguir cinco puntos fundamentales que constituyen la base de la argumentación en LGT: la crítica del hombre económico; los mecanismos de integración económica; el origen y naturaleza del mercado; las mercancías ficticias; y el doble movimiento de la sociedad moderna. Se seguirá el argumento central tratando de explicitar cada uno de estos elementos.

En este sentido, LGT contiene un abierto rechazo de la idea del hombre económico que recorrió el imaginario del pensamiento económico desde Adam Smith hasta el presente. Si bien Polanyi no aborda con detalle las diferencias que, dentro de este campo, se desarrollaron en torno a este problema, adopta una perspectiva histórica lo suficientemente general y primaria como para romper de raíz con cualquier concepción abstracta de la acción económica moderna, capaz de llegar a marcar una distancia básica con el liberalismo del contractualismo como teoría general de la sociedad civil y el Estado (Polanyi, 2003 [1957]:91).

En este marco, es posible trazar el objetivo de LGT como una inversión del problema económico clásico: al contrario, no se trata de suponer el Hombre económico como realidad sustancial de la naturaleza humana en general 
(concepto regulador), sino de comprender el desarrollo y especificidad del hombre moderno y, por lo tanto, la naturaleza de las instituciones que rigen la acumulación de riquezas y la dinámica social (Polanyi, [1957] 2003:94).

Polanyi define una observación problemática primaria: la transformación del lugar y significación del mercado en el mundo moderno respecto de la sociedad premoderna. De ahí que el objetivo específico de su investigación es, por lo tanto, comprender la naturaleza de dicha gran transformación.

De un modo sintético, podría decirse que Polanyi observó que el intercambio mercantil motivado por la ganancia estrictamente pecuniaria, o crematística, ocupó, en las sociedades pre-modernas, un lugar subordinado y de frontera, es decir, ubicado en el límite exterior de la organización social en tanto unidad orgánica.

La centralidad y autonomización del mercado como principio de integración social sólo aparecerá con el desarrollo de la sociedad moderna y con particular intensidad en el siglo XIX. Es esta transformación la que la teoría económica pierde de vista al abstraer al hombre económico como una realidad subjetiva primaria y general, sustancial al salvaje, luego socializado en la relación contractual.

Es importante insistir con el hecho de que Polanyi observa que la institución del intercambio era bastante común desde finales de la Edad de Piedra, aunque sin embargo, su papel era sólo incidental y subordinado en la vida económica (Polanyi, [1957] 2003:91). Frente a esta observación, el autor enfrentó una pregunta obvia que la economía política insistió en desestimar: ¿qué otras relaciones humanas intervienen en la producción y distribución de riquezas? En palabras de Polanyi:

«Pero ¿cómo se asegura [la sociedad antigua] entonces el orden en la producción y la distribución? La respuesta deriva principalmente de dos principios del comportamiento que no se asocian primordialmente con la economía: la reciprocidad y la redistribución» (Polanyi, [1957] 2003:95).

Los términos reciprocidad y redistribución nos introducen el núcleo mismo del pensamiento de Polanyi. En torno a ellos se definen las formas de integración que regulan la producción y distribución de riquezas.

En LGT, Polanyi especifica cuatro mecanismos de integración económica: la reciprocidad, la redistribución, la autarquía familiar y el patrón de mercado. La comprensión de estos mecanismos constituye el paso decisivo para asimilar el sentido del término embeddedness en el pensamiento de Polanyi. Debe notarse al respecto dos aspectos: en primer lugar, cada uno de ellos concurre en la articulación de lo económico, de tal modo que no existe una racionalidad económica primera articulada con otras racionalidades (en oposición al punto de vista de la NSE). A la vez, y en segundo lugar, no existe una estructura abierta e indeterminada de infinitos mecanismos posibles, sino que, por el contrario, lo económico se articula sobre la base de un conjunto limitado y específico de mecanismos característicos (en oposición al punto de vista de crítico de la NSE) que adquieren significación bajo un proceso histórico cultural específico. El paso siguiente es, por lo tanto, mostrar cómo estos mecanismos no se definen en abstracto, sino que, por el contrario, históricamente.

Para abordar este problema puede hacerse una primera distinción. A diferencia del patrón de mercado, el resto de los mecanismos comparten cierta condición 
que, debe reconocérselo, Polanyi no explicita, pero que puede intuirse fácilmente: los tres mecanismos se definen como relaciones sociales de pertenencia, es decir, que nacen de un principio de integración total que se define, no tanto por una sumatoria, como por una identificación de las personas con una totalidad sociocultural. Luego, en un segundo momento, cada uno de estos mecanismos se distingue por sus características formales o funcionales. En el primer caso, la reciprocidad indicará una relación simétrica entre los miembros de una comunidad, mientras que la redistribución indicará una relación asimétrica. Finalmente, el tercer mecanismo de integración, la autarquía familiar, lleva los principios anteriores a la unidad mínima y más próxima de una sociedad determinada, definido por «la producción y el almacenamiento para la satisfacción de las necesidades de los miembros del grupo» (Polanyi, [1957] 2003:101) [las itálicas son nuestras].

Dicho esto, es decisivo no confundir los atributos formales de cada mecanismo (generales y abstractos) con la especificidad histórica que ellos pueden adquirir. Es decir, que los atributos formales pueden ser separados de la especificidad institucional que permite a los sujetos dar significación a la acción económica. Según el propio autor, los mecanismos de integración, «solo pueden volverse eficaces si los patrones institucionales existentes propician su aplicación» (Polanyi, [1957] 2003:96).

Polanyi expresa los atributos formales de los distintos mecanismos en el movimiento de las mercancías. Así, por ejemplo, la reciprocidad se distinguiría por el movimiento simétrico de bienes (o servicios) en el marco de una comunidad definida por unas reglas de restitución; la redistribución, por el contrario, por el movimiento de centralización y luego reparto en torno a una autoridad central; la actividad hogareña por la autarquía en la producción y consumo; finalmente el patrón de mercado por el trocamiento de las mercancías.

Evidentemente, estos movimientos formales no tienen ninguna significación por sí mismos, sino en relación a instituciones históricas en las que se realizan: la comunidad, la autoridad soberana, la familia y el mercado respectivamente.

Hechas estas aclaraciones es preciso avanzar en la definición de estos mecanismos a partir de su especificidad histórica, es decir, bajo un principio que dé inteligibilidad a la gran transformación.

Para ello conviene recuperar la observación problemática introducida por Polanyi: hasta finales del mundo feudal, Europa occidental organizó su vida económica sobre la base de la reciprocidad, la redistribución y la actividad hogareña, sólo marginalmente (en un sentido literal) el mercado ocupó un lugar como mecanismos de articulación: la vida económica estaba estrictamente ligada a la estructura social, es decir, a las diferencias sociales orgánicas que componían la vida en común. La estructura de la comunidad, basada en el mito religioso o mágico, definía los patrones de reciprocidad, redistribución y autarquía, mientras que el mercado tenía un lugar subsidiario articulando el nexo entre la comunidad y lo exterior a ella (Polanyi, [1957] 2003:103).

Polanyi consideraba dos tipos de mercados premodernos: los mercados externos (el intercambio entre diferentes comunidades) y los mercados locales (básicamente los intercambios entre el campo y la ciudad). Sin embargo, aún en estos casos, y particularmente en el segundo, es difícil considerarlos mecanismos 
estrictamente competitivos, es decir, mercados basados en el lucro (Polanyi, [1957] 2003:107).

En línea con lo dicho previamente, Polanyi señala que el mercado es esencialmente distinto de los demás mecanismos ya que no requiere de un vínculo social, previo, total y orgánico. En los otros casos, la simetría, la centralidad y la autarquía, son «meros 'rasgos' y no crean instituciones diseñadas sólo para una función» (Polanyi, [1957] 2003:105). El mercado, por el contrario, se basa en la conjunción entre su función formal y la falta de una relación común. En consecuencia el atributo formal del trocamiento, el mero trocamiento, «es capaz de crear una institución específica: el mercado» (Polanyi, [1957] 2003:106).

La gran transformación se halló, precisamente, en la formación de la economía moderna caracterizada por la emergencia y autonomización del mecanismo de mercado. Este proceso se produjo, según Polanyi, en el marco de una violencia primigenia asociada a la formación del Estado secular y el desarrollo de la mecanización que permitieron, precisamente, la emergencia de mercados de escala nacional. Puesto en un lenguaje espacial Polanyi lo caracterizó como la «extensión del sistema municipal tradicional al territorio más grande del Estado» (Polanyi, [1957] 2003:115).

Es importante notar el carácter cualitativo de la gran transformación. Ella supuso la creación de un nuevo vínculo social orgánico basado en la unidad territorial del estado secular, que abrió el camino a la formación de mercados internos y luego a las transacciones entre ciudadanos de diferentes países. La liberalización mercantil supuso un proceso de secularización (liberándolo del particularismo) de la totalidad orgánica que Polanyi retrata en el doble movimiento de secularización de la unidad político-territorial y de la secularización del intercambio que da lugar a la institución del mercado (Polanyi, [1957] 2003:117).

Esta doble condición podría ser interpretada como una inescindible convivencia de todos los mecanismos de integración, aún bajo el halo de la secularidad moderna: el mecanismo de mercado (basado en una relación de uno a uno) no reemplaza a los mecanismos de redistribución y reciprocidad (basados en una relación de uno al todo), por el contrario, se trata de una secularización de ambos, que inaugura una época histórica caracterizada por una dinámica específica: la vida moderna se manifiesta como un doble movimiento de autonomización del mecanismo de mercado y la recomposición de la unidad orgánica, ambas secularizadas.

El Estado Nación crea en su secularización interna las condiciones del mercado autorregulado, del mismo modo que la disgregación particularista del mercado autorregulado, crea las condiciones de la restauración de la unidad orgánica de las diferencias comunitarias sancionadas en el Estado Nación.

Se trata de un péndulo histórico que se precipita con cambios acelerados entre la autonomización, la crisis y la recomposición de la unidad de un modo permanente y en diferentes escalas.

A fin de comprender este particular movimiento de la vida social moderna Polanyi observó tres mercancías ficticias. Las definió como elementos de la vida social que, en el proceso de autonomización del mercado, suelen ser tratadas como mercancías aun cuando, por sus propias características, no pueden serlo, al menos sin disolver la unidad orgánica de la vida humana. Ello significa 
que, para Polanyi, la crisis sobreviene en la medida en que la autonomización mercantil alcanza y domina sobre estas tres mercancías ficticias: el trabajo (la vida humana misma), la tierra (la naturaleza), y el dinero (la realidad soberana del estado encarnada en el poder de compra que este emite) (Polanyi, [1957] 2003:122-123).

El carácter ficticio de estas mercancías no quiere decir que ellas no sean tratadas en algún momento como mercancías. Por el contrario, el principio de autonomización del mercado produce resultados de transformación social más profundos cuando, precisamente, pretende articularlas como tales. El carácter ficticio se manifiesta, primero, en la naturaleza misma de estos elementos, que son constitutivos de la sociabilidad humana y requieren, por lo tanto, mantener una relación total y orgánica: la vida humana, la relación de hombre con la naturaleza y la unidad de lo común, simplemente se suprimen si la relación total y orgánica se rompe, es decir, si el vínculo primario del uno con el todo se disuelve.

Cuando esta situación se lleva al límite, observa Polanyi, se produce una reacción opuesta que restablece la unidad orgánica, es decir, su vínculo social constitutivo. Polanyi se referirá a lo primero como «dislocación» (Polanyi, [1957] 2003:126) y a la reacción opuesta como la restauración del «hábitat natural del hombre» (Polanyi, [1957] 2003:90).

En el marco de esta dinámica Polanyi llegó a dar inteligibilidad no sólo a la gran transformación que supuso la formación de la sociedad moderna (con su Estado Nación secular y la autonomización del mecanismo de mercado como principio de desarrollo), sino también a la crisis de esta sociedad y a las reacciones que le sucedieron (Unión Soviética, totalitarismos, Segunda Guerra Mundial).

Debe decirse que no es posible hallar en LGT ninguna expresión de adhesión a las formas totalitarias que se levantaron contra la sociedad de mercado, pero sí un intento de comprensión histórica a partir de los propios fundamentos de la sociedad del siglo XIX. Para que ello fuese posible, Polanyi debió desechar, desde un comienzo la noción de que una racionalidad subyacente o un elemento sustancial define la realidad de lo económico, y proponer una fórmula alternativa que él denominó embeddedness según la cual, la producción y el reparto de riquezas se articulan bajo conjunto específico (histórico) de mecanismos de integración económica.

\section{El embeddedness como un proceso institucionalizado}

En trabajos posteriores a LGT, Polanyi vuelve sobre una definición más explícita y sistemática del término embeddedness. Conviene mostrar los rasgos salientes de esta definición a fin de mostrar el hilo argumental que se conserva y que da coherencia al desarrollo de su pensamiento.

En El sistema económicocomo proceso institucionalizado [1957] (1976) Polanyi establece una definición sustantiva de lo económico en la que el embeddedness adquiere pleno significado: la producción y distribución de riquezas debe ser abordada, no en razón de sus aspectos formales, sino a partir de las relaciones sociales que la articulan. En este sentido, el sistema económico se define como un «proceso instituido de interacción entre el hombre y su medio ambiente, que tiene como consecuencia un continuo abastecimiento de los medios materiales que necesitan ser satisfechos» (Polanyi, 1976:159). Los términos subrayados 
luego son destacados por Polanyi. La idea de proceso remite al movimiento, pero aun movimiento muy específico, a saber: el movimiento que rige la apropiación y administración de las cosas de la naturaleza por parte de los hombres, es decir, la actividad que la producción y administración de riquezas supone o, en términos más generales, el movimiento del hombre hacia la naturaleza. Este movimiento de los hombres y las cosas es el que se instituye, es decir, el que se articula bajo ciertas y específicas relaciones sociales.

Luego Polanyi evita explícitamente el camino tomado por la perspectiva abierta de la NSE. Según el propio autor: «En ausencia de cualquier indicación de las condiciones sociales de donde nacen los motivos de los individuos, poco quedaría, si es que algo, que sostuviera la interdependencia de los movimientos y su repetición de que depende la unidad y estabilidad de los procesos» (Polanyi, 1976:160).

Puesto de otro modo, la institucionalización de dicho proceso se produce bajo relaciones que permiten especificarlo históricamente. Polanyi hablará de formas de integración y se refiere, precisamente, a las relaciones sociales específicas que dan estabilidad y unidad, al proceso de acumulación de riquezas. Los dos términos destacados definen el significado del término integración que, en rigor, expresa el argumento clave del enfoque de Polanyi: de lo que se trata, entonces, es de conocer las relaciones sociales (formas) que contienen el proceso, y lo delimitan como sistema, como unidad (integrándolo).

Polanyi, en este trabajo, distingue tres formas de integración fundamentales: el intercambio, la redistribución y la reciprocidad. A fin de definir estas tres formas el autor comienza por una distinción estrictamente formal:

\footnotetext{
«La reciprocidad denota movimientos entre puntos correlativos de agrupamientos simétricos; la redistribución designa los movimientos de apropiación hacia un centro y luego hacia el exterior; el intercambio hace referencia aquí a movimientos viceversa en un sentido y en el contrario que tienen lugar como entre 'manos' en el sistema de mercado» (Polanyi, 1976:162).
}

Sin embargo, el análisis de Polanyi no puede detenerse en este punto. Estas distinciones, formales o abstractas, no dicen demasiado acerca de la especificidad de las relaciones sociales que articulan el proceso de acumulación de riquezas. Por el contrario, el autor va más allá e indica que la reciprocidad es el resultado inmediato de una relación básica: la pertenencia de las personas a una comunidad. Polanyi lo sintetizó de este modo: «Aristóteles enseñó que a toda clase de comunidad (koinonia) corresponde una clase de buena voluntad (philia) entre sus miembros que se manifiesta en forma de reciprocidad (antipeponthos)» (Polanyi, 1976:164).

Es decir que, de un modo más específico, la reciprocidad remite a una identidad común que establece relaciones de reconocimiento (comunidad) y una distribución de roles más o menos simétricos sobre el que se apoyan lazos de solidaridad. Estas relaciones pueden articular procesos de acumulación de riquezas y, por ejemplo, subordinar relaciones de redistribución o intercambio. En todo caso, lo que prima aquí, es el horizonte de significación irreductible de la identificación de un individuo con una comunidad, indispensable para el reconocimiento mutuo que da lugar, luego, a un sistema de normas solidarias. Evidentemente, esta relación es el comienzo de todo lo demás. No hay sociedad posible sin una identidad común mínima que permita dicho reconocimiento. 
En segundo lugar, Polanyi avanza en la especificación de la redistribución. Esta relación sólo puede definirse en el marco de la reciprocidad ya que, precisamente, no es posible pensar en la redistribución sin la existencia de una comunidad previa. La reciprocidad precede a la redistribución aun cuando esta última, eventualmente, parezca independizarse en su desarrollo. Según Polanyi, «la redistribución se consigue, dentro de un grupo, en la medida en que la asignación de los bienes se reúna en una mano y tenga lugar en virtud de la costumbre, la ley o una decisión central ad hoc» (Polanyi, 1976:164). En todo caso, la redistribución podría ser interpretada, en el marco de una comunidad o en razón de ella, como el establecimiento de un centro concentrador ad hoc.

Finalmente, en tercer lugar, sin que medie casualidad alguna, Polanyi aborda los intercambios mercantiles, que precisamente van a definirse por una oposición radical respecto de la identidad con lo común. El mercado, en su forma pura, es la relación de intercambio en la que la cosa intercambiada pesa más que la relación intersubjetiva entre las personas. El intercambio es, en rigor, una relación estrictamente opuesta a la reciprocidad. Por esta razón, entiende Polanyi, es que los mercados permanecieron, en las sociedades pre-modernas, en los márgenes de las comunidades, como pudo verse en el apartado anterior.

A partir de esta oposición sustantiva (reciprocidad vs intercambio) Polanyi puede establecer un principio de inteligibilidad histórica: en toda sociedad pre-moderna, la comunidad, ya sea en su forma simétrica (reciprocidad) o asimétrica (redistribución), o en combinaciones, rigió como el principio societal constitutivo estableciendo un límite dentro del cual, toda relación concreta queda subordinada, mientras que las relaciones mercantiles sólo se ubicaban en los márgenes, es decir, en la relación con otras comunidades, en la administración de bienes poco frecuentes o, directamente, en el espacio de violación de la ley o de la inmoralidad. Por el contrario, en el mundo moderno las relaciones de intercambio mercantil han penetrado el límite de la comunidad y han tendido a ubicarse en el centro mismo del lazo social constitutivo.

Para definir con mayor claridad esta última idea Polanyi indica, finalmente, que «el predominio de una forma de integración se identifica aquí con el grado en que abarca la tierra y el trabajo de la sociedad» (Polanyi, 1976:166). A partir de allí la reconstrucción del hilo argumental de LGT ya no supone mayores problemas.

La construcción del espacio nacional moderno supuso, simultáneamente, la formación de un mercado nacional. Estas dos instituciones seculares abrieron paso a una dinámica doble de autonomización del mercado, avanzando sobre las mercancías ficticias y de restauración de la comunidad orgánica. Crisis en medio de la cual el propio Polanyi vivió e intentó retratar de un modo inteligible.

\section{A modo de cierre}

Como pudo verse Polanyi estableció en sus trabajos posteriores, los principales conceptos desarrollados en LGT. En dicha continuidad es posible separar el argumento sintético que da la unidad conceptual al enfoque del autor: Polanyi define lo económico por las formas de integración que articulan la producción y distribución de riquezas. Estas formas de integración parten de una tensión histórica, dinámica e indisoluble entre la unidad orgánica de la 
comunidad (reciprocidad y redistribución) y su inversión: la individualidad (del intercambio). Luego, la sociedad moderna se define como aquella etapa en la que esta tensión pasó por el cristal de la secularización y se precipitó sobre la autonomización del mercado, cuyo desarrollo no hizo más que invocar, de un modo permanente, una reacción en contrario: una restauración de la unidad dislocada.

Esta noción de lo económico y, por lo tanto, del término embeddedness, es significativamente diferente de cualquier versión expuesta en el marco de la NSE, ya se trate de la corriente principal y como los enfoques críticos. La diferencia es explícita una vez conocido el argumento central: no existe una racionalidad de última instancia equivalente con lo económico, ni lo económico es resultado de una estructuración a priori vacía. Por el contrario, el enfoque de Polanyi se apoya en una consideración ontológica clave: la sociedad debe ser interpretada como una unidad sociocultural con diferencias internas (orgánica), lo que supone una tensión permanente en razón de su propia naturaleza. En el mundo moderno esta tensión, entre unidad y diferencia, se radicaliza en la autonomización del mercado: el individuo queda frente a la comunidad como en una relación de exterioridad, pero, simultáneamente, también se disuelve en ella como una unidad indiferenciada. Polanyi revela de este modo una dinámica de la vida social moderna que, por su parte, permite explicar, comprendiendo, el sentido de la acción económica bajo las formas de integración de las que el autor parte y que, además, fueron resultado de la observación histórica y antropológica de largo plazo.

\section{Referencias}

BECKERT, JENS (1996): «What is sociological about economic sociology? Uncertainty and the embeddedness of economic action», en: Theory and society, 25, 6, pp. 803-840.

BECKERT, JENS (1999): Economic Action and Embeddedness: The Problem of the Structure of Action. UC Berkeley: Center for Culture, Organizations and Politics - Previously Affiliated. Disponible en https://escholarship.org/uc/item/6jc6f1q6

BECKERT, JENS (2003): «Economic sociology and embeddedness: how shall we conceptualize economic action?», en: Journal of Economic Issues, 37, 3, pp. 769-787.

BECKERT, JENS (2007): «The Great Transformation of Embeddedness: Karl Polanyi and the New Economic Sociology», MPIfG Discussion Paper, 07/1.

CAMPREGHER, GLAUCIA; SCHÖNHOFEN LONGONI, LUCAS (2017): «A natureza humana do comportamento individual nos primórdios do pensamento econômico: uma comparação entre Hume, Smith e Bentham», en: Economia e Sociedade, 26, 1, pp. 111-139.

CONVERT, BERNARD; HEILBRON, JOHAN (2007): «Where did the new economic sociology come from?», en: Theory and Society, 36, 1, pp. 31-54.

GRANOVETTER, MARK (1973): «The Strenght of Weak Ties», en: American Journal of Sociology, 78, 6, pp. 1360-1380.

GRANOVETTER, MARK (1983): «The strength of weak ties: A network theory revisited», en: Sociological Theory, 1, 1, pp. 201-233. 
GRANOVETTER, MARK (1985): «Economic action and social structure: The problem of embeddedness», en: American Journal of Sociology, 91, 3, pp. 481-510.

GRANOVETTER, MARK (1990): «The old and the new economic sociology: A history and an agenda», en: Friedland, Roger y Robertson, A. F. (eds.). Beyond the marketplace: Rethinking economy and society, New York, Aldine de Gruyter, pp. $89-112$.

GRANOVETTER, MARK (2000): «La fuerza de los vínculos débiles», en: Politica y sociedad, 33, pp. 41-56.

GRANOVETTER, MARK (2017): Society and economy. Harvard University Press.

GRANOVETTER, MARK; SWEDBERG, RICHARD (eds.). (2001): The sociology of economic life, Boulder, Westview press.

KRIPPNER, GRETA (2002): «The elusive market: Embeddedness and the paradigm of economic sociology», en: Theory and society, 30, 6, pp. 775-810.

NEE, VICTOR; INGRAM, PAUL (1998) «Embeddedness and Beyond: Institutions, Exchange, and Social Structure», en: Brinton, Mary y Nee, Victor (eds.). The New Institutionalism in Sociology, Stanford, CA: Stanford University Press, pp. 19-45.

PARSONS, TALCOTT; SMELSER, NEIL [1956] (2005): Economy and Society. A Study in the Integration of Economic and Social Theory, London, Taylor \& Francis.

POLANYI, KARL [1957] (1976): «El sistema económico como proceso institucionalizado», en: Godelier, Maurice. (ed.). Antropología y economía, Barcelona: Anagrama, pp. 155-178.

POLANYI, KARL [1957] (2003): La gran transformación: los origenes politicos y económicos de nuestro tiempo, México, Fondo de Cultura Económica.

PORTES, ALEJANDRO (2010): Economic sociology: a systematic inquiry, Princeton, Princeton University Press.

SMELSER, NEIL (1964): Sociología de la vida económica, México, Unión Tipográfica Editorial Hispano Americana.

SMELSER, NEIL; SWEDBERG, RICHARD (eds.). (2010): The handbook of economic sociology, New York, Russell Sage Foundation y, Princeton, Princeton University press.

SWEDBERG, RICHARD (1991): «Major traditions of economic sociology», en: Annual Review of Sociology, 17, 1, pp. 251-276.

SWEDBERG, RICHARD (2001): «Hacia una nueva sociología económica. Balance y perspectivas», en: Trabajo, 2, 4, pp. 5-34.

SWEDBERG, RICHARD (2005): Max Weber e a idéia de sociologia económica, Rio de Janeiro, Editora Universidade Federal do Rio de Janeiro.

SWEDBERG, RICHARD; HIMMELSTRAND, ULF; BRULIN, GÖRAN (1987): «The paradigm of economic sociology: premises and promises», en: Theory and society, 16, 2, pp. 169-213.

UZZI, BRIAN (1997): «Social structure and competition in interfirm networks: The paradox of embeddedness», en: Administrative Science Quarterly, 42, 1, pp. 35-67.

WALDINGER, ROGER (1995): «The 'Other Side' of Embeddedness: A Case Study of the Interplay between Economy and Ethnicity», en: Ethnic and Racial Studies, 18 , pp. 555-80.

ZELIZER, VIVIANA (1988): «Beyond the polemics on the market: establishing a theoretical and empirical agenda», en: Sociological forum, 3, 4, pp. 614-634.

ZELIZER, VIVIANA (2008): «Pasados y futuros de la sociología económica», en: Apuntes de Investigación del CECYP, 14, pp. 95-112. 
ZELIZER, VIVIANA (2010): Economic lives: How culture shapes the economy, Princeton, Princeton University Press.

ZUKIN, SHARON; DIMAGGIO, PAUL (eds.) (1990): Structures of capital: The social organization of the economy, New York, Cambridge University Press.

\section{Notas}

[1] Puede consultarse la versión ampliada en castellano en Granovetter (2000).

[2] Campregher y Longoni (2017) analizan el problema del comportamiento en Hume, Smith y Bentham y recaban precisamente sobre la distancia que los separa de la filosofía moral y política del utilitarismo que posteriormente va formalizar la teoría marginalista 